\title{
Tolerability and acceptability of Blephagel: a novel eyelid hygiene aqueous gel
}

This article was published in the following Dove Press journal:

Clinical Ophthalmology

10 January 2012

Number of times this article has been viewed

\section{Serge Doan}

Department of Ophthalmology, Bichat Hospital and Foundation, Paris, France

Correspondence: Serge Doan

Department of Ophthalmology,

Bichat Hospital and Foundation,

46 rue Henri Huchard, 75018 Paris,

France

Tel +33 I 40258443

Fax +33 । 40258838

Email serge.doan@noos.fr
Abstract: Commonly associated with dry eye, blepharitis is the most commonly encountered disorder in general ophthalmologic practice. Although anti-infective and anti-inflammatory therapies are available, eyelid hygiene is the cornerstone of effective management. A variety of products have been used to assist and encourage eyelid cleansing and massage. The present study examines the ocular and periocular tolerability and acceptability of Blephagel, a cosmetic, poloxamer-containing gel designed specifically for cleansing the eyelid in subjects with sensitive skin or eyes or contact lens users. Subjects with blepharitis and sensitive skin or eyes, a history of atopy, or who use contact lenses applied Blephagel twice daily at home. Clinical ophthalmologic examinations were conducted before and 21 days after aqueous gel application, and subjects completed a questionnaire on the acceptability of the preparation. Thirty-three predominantly female subjects entered and completed the study. A total of $36 \%$ of the subjects had used similar products in the past, $21 \%$ regularly. Upon questioning by the ophthalmologist, $85 \%$ of the subjects reported acceptability of the preparation as good to very good, and $73 \%$ rated the efficacy as good to very good. There were minor but statistically nonsignificant changes in fluorescein tear breakup time and visual acuity before and after 21 days of aqueous gel application. The questionnaire results indicated that the subjects found the product to be effective for cleansing the eyelids of mucus and squama around eyelash roots. Moreover, cosmetic qualities, sensation in use, and acceptability were also appreciated. No subject reported any adverse event considered to be related to the aqueous gel. Although the safety of Blephagel has already been established in standard tests, the current results suggest that it is also pleasant to use and acceptable to blepharitis patients with sensitive skin as an aid to an eyelid hygiene regime.

Keywords: blepharitis, tolerability, eyelid hygiene

\section{Introduction}

Blepharitis is a common disorder. ${ }^{1}$ Indeed, it is the most common disorder encountered in general ophthalmologic practice ${ }^{2}$ and is characterized by inflammation of the eyelid margin; sore eyelids; irritated, burning, or gritty sensations in the eye; dry, watery, or irritated eyes; sensations of foreign body in the eye; or eyelids sticking together in the morning. It is commonly associated with dry eyes. As well as being highly distressing for the patients, it is a risk factor for endophthalmitis following cataract surgery. ${ }^{3,4}$ Blepharitis can be posterior, anterior, or mixed. Posterior blepharitis involves meibomian glands and is related to meibomian gland dysfunction. Anterior blepharitis involves the anterior lid margin and eyelashes and is typically associated with staphylococcal infection or seborrhea and, for some, infection by Demodex. ${ }^{5,6}$ 
The pathophysiology of blepharitis is complex and involves several different interacting systems, including secretions of the lid margin, disorder of the tear film, and infection of the lid margin. The structure of the lid margin is crucial to the health of the eyelid; the meibomian glands secrete lipids, which spread themselves on the tear film to prevent excess tear evaporation. A reduction in the quality or quantity of the meibomian gland secretions can result in a poor-quality tear film, an increased rate of evaporation, dry eyes, and blepharitis. Disorders of the meibomian glands may be primary or secondary to skin disorders such as rosacea or seborrheic dermatitis. Infectious agents can interact with meibomian secretions and promote, propagate, and perpetuate meibomian gland dysfunction, tear film instability, and ocular surface inflammation. ${ }^{7}$

The pathologic meibomian and sebaceous secretions in meibomian gland dysfunction contain irritative lipids like free fatty acids and fatty acid peroxides that promote inflammation of the lid margin and should be cleaned off the lid margin. The colarettes are a shelter for Demodex mites, which are very frequently found in eyelashes with colarettes. ${ }^{8}$ Mechanical removal of these crusts and collarettes is thus an important step of lid hygiene. Although soap and mild detergents are effective, they are often irritative and might have a negative effect on the ocular surface, especially on the tear film.

Eyelid hygiene has an important role to play in the prevention and treatment of blepharitis. This includes not only cleaning of the eyelid but also encouraging lid warming and specific massage manoeuvers to promote meibomian gland secretion and the removal of crusts from the eyelashes and eyelid margin. The mechanism of action of such eyelid cleaning procedures and massage appears to be multifactorial and includes thickening and stabilization of the meibomian lipid layer ${ }^{9}$ (partly, at least, by manual stimulation and expression of the meibomian glands) as well as reducing bacterial colonization, which has deleterious effects on the meibomian lipid layer. ${ }^{10} \mathrm{~A}$ variety of products (in the form of shampoos and scrubs formulated specifically for eyelid hygiene) exist to assist in the cleaning/massage routine and appear to be effective, ${ }^{11}$ some commercially formulated cleansers being less irritating than soap or shampoo. ${ }^{12}$ Patients appear to favor such products over simple soap or diluted shampoos, ${ }^{13}$ and their use may improve compliance with eyelid hygiene regimes. Interestingly, studies suggest that an eyelid hygiene regime not only reduced preoperative conjunctival flora ${ }^{14}$ but also was effective in reducing the number of cataract surgery cancellations due to blepharitis. ${ }^{15}$

Although pharmacologic approaches, including antibacterial, anti-inflammatory medication, can be useful in particular cases, the mainstay of treatment and prevention in blepharitis is rigorous eyelid hygiene. There is considerable scope for products that not only provide effective cleaning of the eyelid and lid margin but are more convenient and pleasant in everyday use. Such products should be acceptable to blepharitis patients, who are often sensitive to the application of such products because of their sensitive skin and eyes. Indeed, compliance with eyelid hygiene may be poor in such patients because of intolerance to topical treatments.

Blephagel (Laboratoires Thea, Clermont-Ferrand, France) is a cosmetic, poloxamer-containing gel designed specifically for cleansing the eyelid that is currently undergoing development as an aid to eyelid hygiene. Blephagel has a soothing action that encourages routine use and leaves the skin nongreasy. Rinsing is not required, so the product may be used away from the bathroom or when traveling.

The safety and tolerability of Blephagel has been demonstrated in in vitro and in vivo studies in healthy volunteers. ${ }^{16,17}$

Although the in vitro and in vivo data are encouraging, to date there have been no studies on the tolerability and acceptability of Blephagel in the more sensitive environment of the eyelid and periocular region, nor among subjects who might be expected to be particularly sensitive to the application of the product around the eye.

The objective of the current study was to determine the ocular and periocular tolerability of Blephagel in subjects with sensitive skin or eyes, contact lens users, or those with a personal or family history of atopy.

\section{Methods}

This open study without comparator was undertaken at a single center in France. Although the investigational product is classified as a cosmetic, the study was conducted in the spirit of the most recent recommendations of the World Medical Association (Declaration of Helsinki 1964; last amendment in force), Good Clinical Practice, and International Conference on Harmonisation of Technical Requirements for Registration of Pharmaceuticals for Human Use (Topic E6: CPMP/ICH/135/95), and in accordance with relevant French legislation and the standard operating procedure of Institut d'Expertise Clinique, Lyon, France, insofar as these were applicable to the type of product.

\section{Investigational product}

Blephagel is a colorless, aqueous gel comprising carbomer, poloxamer 188 , macrogol 4000, sodium hydroxy methyl glycinate, sodium hydroxyde $1 \mathrm{~N}$, and purified water, 
presented in an airless plastic pump flask for administration by the subject. The product was applied twice daily for 3 weeks.

\section{Participants}

Selection of subjects from a panel was made by an ophthalmologist investigator by means of a prestudy questionnaire and medical examination based on the inclusion and exclusion criteria.

\section{Inclusion criteria}

Healthy 18 to 70-year-old Caucasian men or women able and willing to give informed consent who identified themselves as having sensitive skin of the eye contour and eyelid and who were either contact lens users or had a personal or family history of atopy were eligible for inclusion.

Subjects with sensitive skin were those who reported abnormal and repeated reactions of the skin (tightness, prickling, itching, redness) to face care products, hygiene products (including soap), the environment during a major part of the year, or other factors such as water, clothes, or shaving.

Sensitive eyes were defined to include frequent prickling of the eyes, episodic watering, or becoming red in contact with environmental factors. Reaction to at least one of wind, cold, sun, and allergens and/or at least three of light; water (shower, swimming pool); ocular pain when watching television, reading, driving, or using a computer; use of cosmetics; or exposure to pollution. Fear of exposure to sunlight and constant wearing of sunglasses was not, in itself, an inclusion criterion.

A personal history of atopy was defined as a history of constitutional eczema, mostly appearing during childhood and mostly located in the skin folds; recurrent periodic asthma in childhood or preteenage years (no asthma crisis should have occurred during the last 6 months); recurrent periodic (chronic) conjunctivitis; or pneumallergen-related allergic rhinitis. A family history of atopy was defined as at least two parents or siblings with a history of atopic dermatitis, allergic asthma in the first half of life, hay fever, or dermo-respiratory syndrome.

\section{Exclusion criteria}

The health status of subjects was verified by the investigator by means of a detailed history, with particular attention to gynecologic, medical, surgical, and cutaneous conditions as well as vaccination status and smoking habits. The following categories of subjects were excluded from the study:
- Pregnant or breastfeeding mothers, or subjects not using a medically acceptable contraceptive method; subjects having undergone organ excision, organ transplant, or skull concussion with extended loss of consciousness; and subjects with a known medical history, disorder, or disease and/or any complicating factor or structural abnormality judged by the investigator to be incompatible with the study;

- Subjects

- who had undergone ocular surgery or on longterm treatment, in particular with antihistaminics, steroids, beta blockers (including eye lotion), and/or desensitization

- with a history of drug intolerance or allergy to products for professional use

- with a skin disease

- with a disease of the immune system or on immunosuppressive treatment or having had an asthma crisis during the last 6 months

- who smoke more than the equivalent of ten cigarettes a day or consume more than the equivalent of two glasses of wine per day, or those exhibiting drug abuse and/or excessive use of medications

- currently involved in another biomedical research project

- who had an asthma crisis during the last six months

- with macroscopic traces of irritation or any other abnormality on the areas of product application that could interfere in the analysis of the results or having taken, in the past 3 months, medical treatment that is, in the ophthalmologist investigator's judgment, inconsistent with the participation in the study and thus makes $\mathrm{him} /$ her ineligible, in particular for anti-inflammatories applied on the test area within the 2 weeks before the beginning of the study and corticoids during the month before the beginning of the study

- currently receiving anti-allergy injections, with a final injection within the last 8 days (except lag effect corticoid injection for which the last one must be 6 months before), or expecting to begin injections during the study

- with a febrile illness

- who had been vaccinated in the month preceding the start of the study or expecting to be vaccinated during the study

- who had modified their cosmetic habits in face cleaning during the last 2 weeks or who had applied a cosmetic product on the areas concerned by the study on the inclusion day of the study 
- having skin recently exposed to sunlight (natural or artificial) or having had heliotherapy in the last 2 weeks

- who had undergone surgery on the concerned areas (face, eye contours) or chemical or physical treatment to the face (eg, peeling, injections).

Other medication during the study or in the week before (excepting paracetamol and oral contraceptives) was not permitted and constituted grounds for exclusion. Protocol violations or emergence of exclusion criteria were also grounds for withdrawal from the study.

\section{Product application}

Subjects were instructed on the appropriate method of application of the product in which a single pump of product (representing a mean volume of $0.46 \mathrm{~mL}$ ) was to be applied on nonsterile, nonwoven compresses given to the subject for 3 weeks, by gentle massage to the eyelids, eyelashes, and around the orbit. Remaining product was to be removed by compress. Subjects were advised not to apply the product into the eye.

\section{Outcome measures}

\section{Ophthalmologic examination}

On the first and last day of the study, subjects underwent a structured ophthalmologic examination in which the state of the eyelids, palpebral margin and eyelashes, tarsal or bulbar conjunctiva, cornea, anterior chamber, direct and consensual photomotor reflexes, and fluorescein tear breakup time were rated on predefined ordinal scales. In addition, measurement of visual acuity was performed.

The right and left eyes of each subject were examined by the ophthalmologist investigator to evaluate the condition of the eyelids on macroscopic examination quantified according to a $0-4$ scale $(0=$ normal; $1=$ slightly red skin; $2=$ red and edematous skin; $3=$ lid eczema; $4=$ desquamation). Palpebral margin and eyelashes were examined using a slit lamp (Nikon Corporation, Shinjuku, Japan, Zoom photo, Slit lamp FS 3$)$ and rated according to a $0-3$ scale $(0=$ normal; $1=$ local redness on the palpebral margin only; $2=$ secretions and crusts on the eyelashes; 3 = falling eyelashes, considerable blepharitis). The state of the tarsal or bulbar conjunctiva was evaluated using a slit lamp according to a $0-4$ scale $(0=$ normal; $1=$ slight redness [vasodilatation visible only with the slit lamp]; 2 = clearly visible redness [clear vasodilatation visible macroscopically]; $3=$ some small follicles and papillae; $4=$ numerous follicles and large papillae). Corneal condition was evaluated by slit lamp according to a $0-4$ scale $(0=$ normal; $1=$ slight localized cellular alterations; 2 = clear and spread cellular alterations; 3 = superficial punctuate keratitis on the whole cornea; $4=$ corneal ulcer and edema). The anterior chamber was evaluated according to a $0-3$ scale $(0=$ normal; $1=$ tyndall; 2 = full-blown iritis or descemetitis; $3=$ hypopion). Direct and consensual photomotor reflexes were evaluated by ophthalmoscope lamp according to a $0-2$ scale $(0=$ normal; $1=$ slow; $2=$ nil). Fluorescein tear film breakup time value was scored in seconds as the average of the two measurements, with a third measurement taken in case of discrepancy $>2$ seconds.

The nature and sensitivity of the subject's facial and eye contour/eyelid skin was also evaluated at the first visit using the following categorization: normal, mixed oily, oily, mixed dry, dry, and very dry.

The subjects were questioned regarding the acceptability and efficiency of the product by means of a questionnaire designed for "in-use test under ophthalmologic control" and validated by the research organization responsible for the study. The questionnaire was applied by the investigating ophthalmologist.

\section{Functional signs}

On the last day of the study the ophthalmologist investigator assessed the degree of discomfort felt by the subject in terms of symptoms of palpebral pruritus, ocular prickling, transitory heat, ocular pain, eye watering, discomfort to light, transitory mist, palpebral redness, palpebral desquamation, palpebral swelling, eyelash involvement, secretions, and ocular redness on a 4-point ordinal scale $(0=$ no functional signs; 1 = very slight discomfort; 2 = notable discomfort; 3 = symptoms sufficiently serious to limit the subject's daily activities).

\section{Effect on contact lenses}

On the last day of the study, examination was undertaken of contact lenses according to deposits, coloration, opacification, variation in mobility, and changes in the suppleness of lenses on a 4-point ordinal scale $(0=$ absence of reaction; $1=$ start of reaction; $2=$ moderate reaction; $3=$ severe reaction).

\section{Acceptability and efficiency questionnaire}

Acceptability and efficiency (as a "cleaning product for eyelids and eyelashes") of the product was assessed by a selfadministered questionnaire (on a 5-point ordinal scale: nil, 
poor, fairly good, good, very good) and an overall appraisal given verbally to the investigator.

\section{Adverse events}

Adverse events, if any, were reported by the subjects. In addition, the examining ophthalmologist identified changes in the medical status of the subjects between the first and final visit.

Subjects were withdrawn from the study upon emergence of adverse events considered to be linked to the study product.

\section{Results}

\section{Subject disposition}

Fifty-six subjects were recruited, of whom 37 attended the clinic for the first visit. Thirty-three were then entered into the study. All subjects returned at the final visit and were evaluable for acceptability and safety.

\section{Subject characteristics}

The subjects were predominantly female $(85 \%)$ with a mean age of 47.8 years (range 20-69 years). The nature of the subject's facial skin was evaluated as normal $(0 \%)$, mixed oily (30\%), oily ( $0 \%)$, mixed dry ( $45 \%)$, dry $(21 \%)$, and very dry $(3 \%)$. All subjects were determined to have sensitive facial skin. The majority (64\%) of subjects had dry skin of the eye contour and eyelid, with $33 \%$ and $3 \%$ having dry or very dry skin of the eye contour and eyelid, respectively. Again, all subjects had sensitivity of the skin of the eye contour and eyelid.

Of the subjects, $76 \%$ had sensitive eyes, $61 \%$ were contact lens wearers, and $39 \%$ had a personal or family history of atopy. A total of $36 \%$ were habitual users of the products similar to the investigational product, with $21 \%$ being regular users.

\section{Ophthalmologist assessment}

There were minor changes in the lachrymal film breakup time and visual acuity between the first and last visits, but neither achieved statistical or clinical significance (Figure 1). The visual acuity measurements obtained before and after the 3 weeks of application evolved within clinically acceptable limits.

\section{Safety and tolerability}

Of the subjects, $85 \%$ reported no ocular or periocular signs or symptoms. Of the five subjects who reported clinical

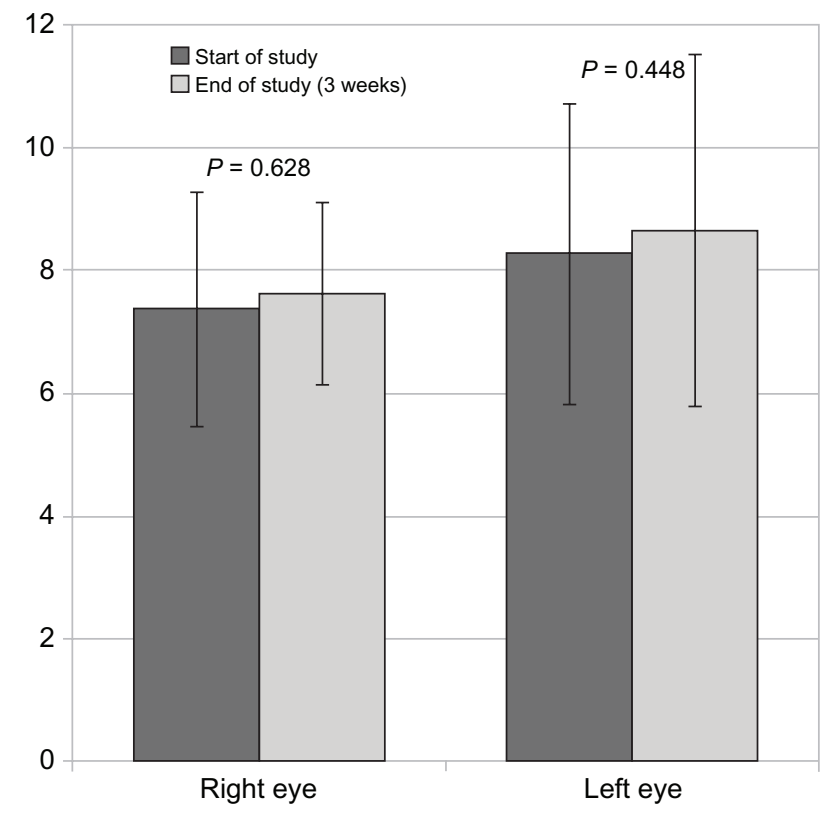

Figure I Lachrymal film breakup time before and after 3 weeks of Blephagel use (mean of three measures in 13 subjects). Lachrymal film measured by breakup time scored in seconds. The breakup time value was scored as the average of the two measurements, with the third measurement taken in case of discrepancy $>2$ seconds.

signs and symptoms, there were four reports of prickling, two reports of eye watering, and one each of palpebral swelling and tightness. Four reports of symptoms were attributed to a single subject. None of the adverse events was linked to the investigational product, and none led to withdrawal.

\section{Acceptability and efficiency}

Among the seven subjects who were habitual users of similar products, six assessed the investigational product as being as well tolerated as their usual product. The remaining subject reported their usual product as being better tolerated.

Following questioning by the investigating ophthalmologist, $85 \%$ of subjects rated the acceptability and $73 \%$ rated the efficiency of the product as good to very good (Figure 2).

\section{Appraisal by the subject}

The questionnaire responses are shown in Figure 3.

Of the subjects, $85 \%$ considered that "the product is ideally suitable for the cleansing of the eyelids, the mucus, and the squama that "clutter' the eyelash roots."

Moreover, the product was also appreciated for:

- its cosmetic qualities ("good enough", consistency (85\%), "good enough texture" (88\%), "easy application" (94\%), "does not leave the skin oily" (94\%)/"sticky" (91\%), "does not leave any traces" (94\%) 

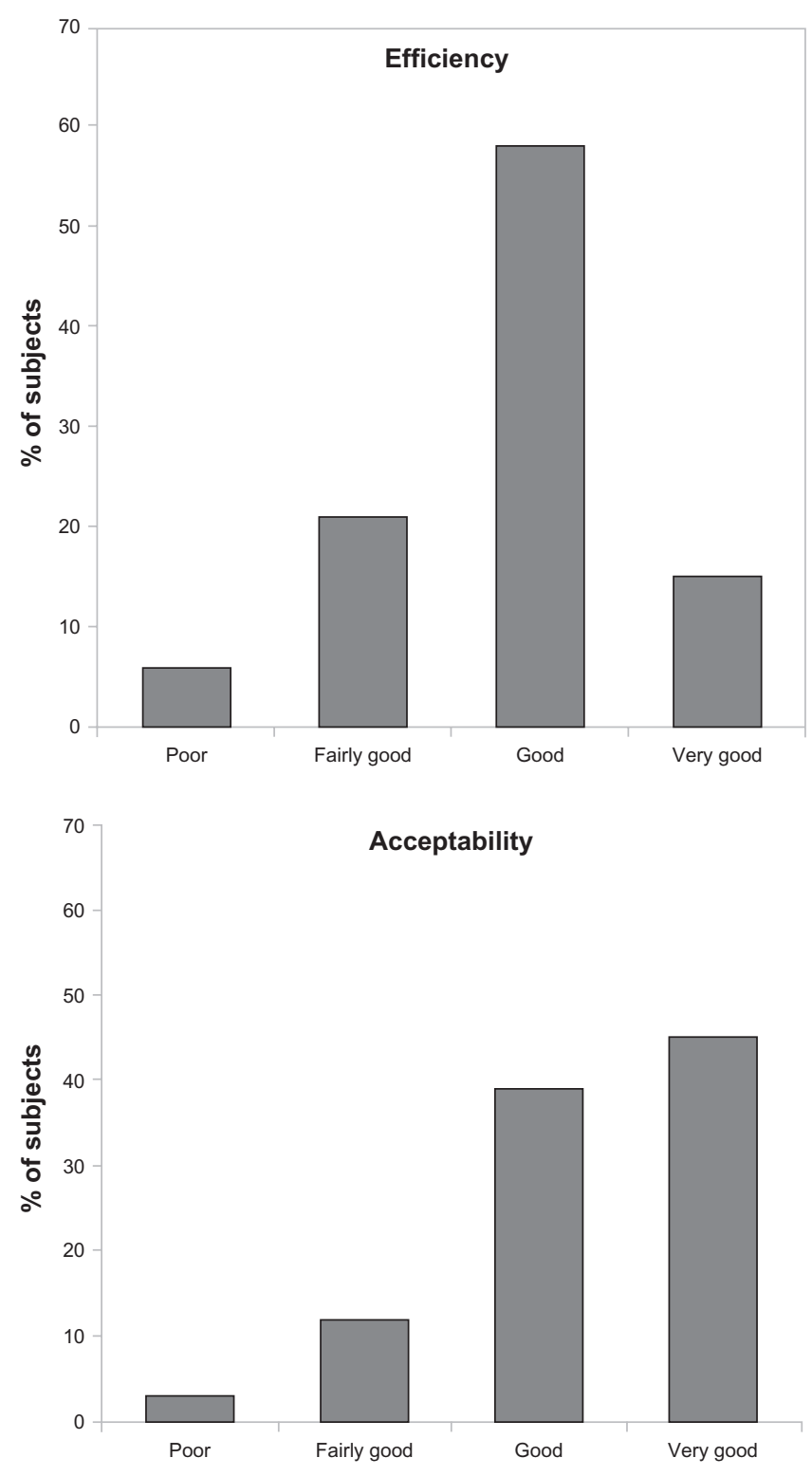

Figure 2 Appraisal of efficiency and acceptability by subjects in the presence of the investigating ophthalmologist. Acceptability and efficiency (as a "cleaning product for eyelids and eyelashes") of the product was assessed by a self-administered questionnaire (on a 5-point ordinal scale: nil, poor, fairly good, good, very good).

- the felt sensations ("gives a pleasant sensation of freshness" (85\%), "softens" (79\%), "soothes the eyelids" (79\%), "leaves the eyelids comfortable" (88\%)

- its acceptability ("suitable for the subjects skin type" (97\%), “for sensitive skins" (97\%)/“eyelids” (94\%).

\section{Discussion}

Hygiene of the eyelids is crucial to the prevention of blepharitis. The present study has further indicated that Blephagel is well accepted and appreciated by subjects with risk factors for susceptibility (the majority of whom had multiple risk factors) themselves. Acceptability and efficacy were rated as good or very good by a large majority of the subjects. Ophthalmologic examination revealed no concerns, and the number of subjects reporting ophthalmologic signs was very low. There were no reports of adverse events linked to Blephagel.

The safety and tolerability of Blephagel have been demonstrated in in vitro and in vivo studies. Blephagel has been evaluated in the standard battery of tests for cosmetic products intended to be used around the eyes and has been shown to be safe and nonirritant. Although not intended for application in the eye, Blephagel has been tested in the isolated bovine cornea opacity and permeability test ${ }^{16}$ for ocular irritation and was rated in the lowest possible category for ocular irritation (manufacturer data on file). The bovine cornea opacity and permeability test is a standard in vitro technique in which the penetration of a fluorescein dye into bovine corneas in vitro is assessed. In an in vivo skin tolerability test involving 48 hours of continuous exposure to the skin on the back of ten human subjects, no signs of erythema, edema, papulae, vesicles, bulbae, or pustules were observed, nor evidence of dryness, desquamation, detergent effect, or reflectivity. The primary cutaneous irritation index calculated in this study was 0 (manufacturer data on file).

A further study, the so-called final clinical safety test, was carried out to confirm the good cutaneous compatibility and the absence of delayed cutaneous sensitizing potential in healthy adult subjects. Using the method of Marzulli and Maibach, ${ }^{17}$ with single and repeated epicutaneous applications of the investigational product to elicit delayed reaction, the final clinical safety test similarly revealed no primary or cumulative irritation reactions, nor any cutaneous sensitization to Blephagel, among 107 exposed subjects (manufacturer data on file). Further controlled clinical studies in larger groups of patients would be welcome to confirm these results.

Despite widespread acceptance of eyelid hygiene as the most effective means of treatment and prevention of blepharitis, formal studies are scarce, though studies have indicated that such regimes improve the quality and thickness of meibomian gland secretions in subjects with disorder of the meibomian glands. ${ }^{18,19}$

The present study shows Blephagel to be well tolerated and accepted in a subject population who could be considered to be particularly sensitive to the application of such products. Blephagel may be a useful and convenient addition to the daily eyelid hygiene regime of blepharitis sufferers.

\section{Acknowledgments}

The study was undertaken by IEC France, 88, boulevard des Belges, 69006 Lyon, France, on behalf of the manufacturer 


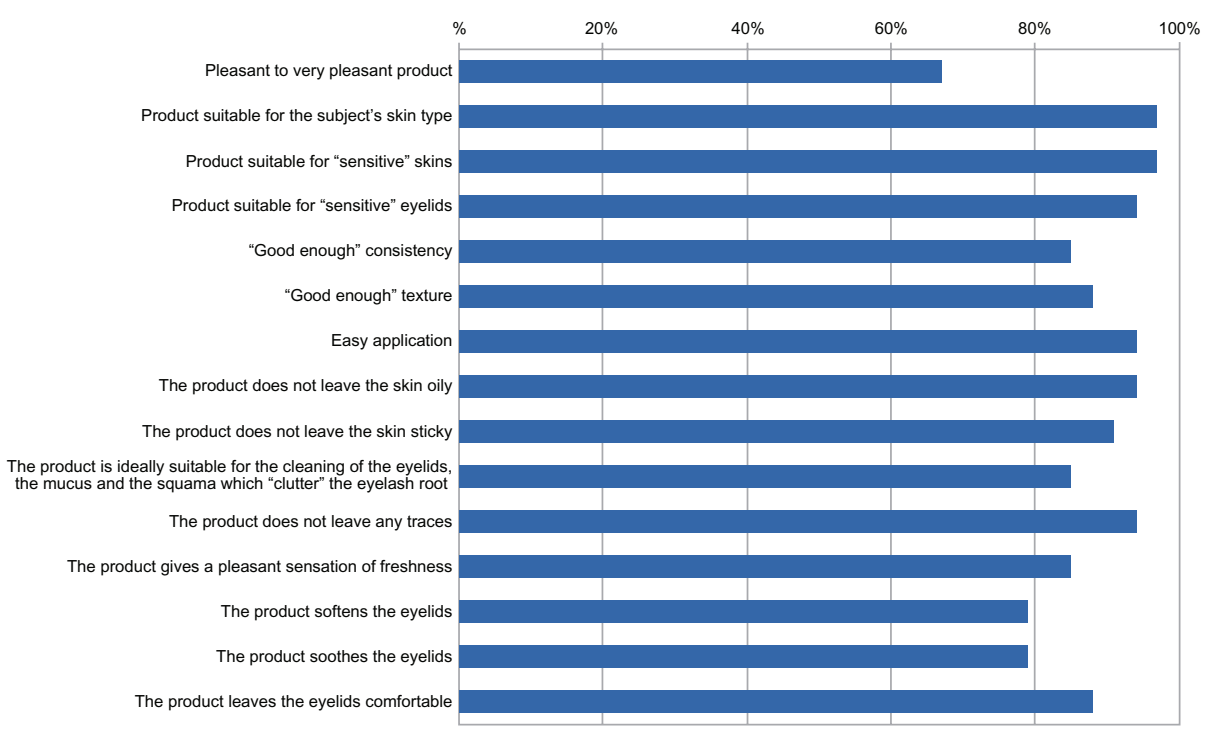

Figure 3 Subject appraisal of the investigational product qualities and efficacy.

Laboratoires Thea, 12, rue Louis Blériot 63017 ClermontFerrand, France. Assistance in the preparation of the manuscript was provided by JSI Communications Ltd, Sunart, Eccles Lane, Whaley Bridge, UK.

\section{Disclosure}

Dr Doan is a consultant to Thea Laboratories. The authors report no conflicts of interest in this work.

\section{References}

1. Lemp MA, Nichols KK. Blepharitis in the United States 2009: a surveybased perspective on prevalence and treatment. Ocul Surf. 2009; 7 Suppl 2: S1-S14.

2. Benitez del Castillo Sanchez JM, Del Rio Novo MT, Garcia-Sanchez J. Frecuencia de la blefaritis en la consulta oftalmologica diaria. St Ophthal. 1999; XVIII (3):225-230.

3. Sparrow JM. Monte-Carlo simulation of random clustering of endophthalmitis following cataract surgery. Eye. 2007;21:209-213.

4. Speaker MG, Milch FA, Shah MK, Eisner W, Kreiswirth BN. Role of external bacterial flora in the pathogenesis of acute postoperative endophthalmitis. Ophthalmology. 1991;98:639-649.

5. Jackson WB. Blepharitis: current strategies for diagnosis and management. Can J Ophthalmol. 2008;43:170-179.

6. Smith RE, Flowers CW. Chronic blepharitis: a review. CLAO J. 1995;21: 200-207.

7. Nichols KK. The international workshop on meibomian gland dysfunction: introduction. Invest Ophthalmol Vis Sci. 2011;52:1917-1921.

8. Gao YY, Di Pascuale MA, Li W, et al. High prevalence of Demodex in eyelashes with cylindrical dandruff. Invest Ophthalmol Vis Sci. 2005 ; 46:3089-3094.

Clinical Ophthalmology

\section{Publish your work in this journal}

Clinical Ophthalmology is an international, peer-reviewed journa covering all subspecialties within ophthalmology. Key topics include: Optometry; Visual science; Pharmacology and drug therapy in eye diseases; Basic Sciences; Primary and Secondary eye care; Patient Safety and Quality of Care Improvements. This journal is indexed on Submit your manuscript here: http://www.dovepress.com/clinical-ophthalmology-journal
9. Gilbard JP. The diagnosis and management of dry eyes. Otolaryngol Clin North Am. 2005;38:871-885.

10. International Dry Eye Workshop. Management and therapy of dry eye disease: report of the Management and Therapy Subcommittee of the International Dry Eye WorkShop (2007). Ocul Surf. 2007;5: 163-178.

11. Polack FM, Goodman DF. Experience with a new detergent lid scrub in the management of chronic blepharitis. Arch Ophthalmol. 1988; 106:719-720.

12. Leibowitz HM, Capino D. Treatment of chronic blepharitis. Arch Ophthalmol. 1988;106:720.

13. Key JE. A comparative study of eyelid cleaning regimens in chronic blepharitis. CLAO J. 1996;22:209-212.

14. Hueso Abancens JR, Mengual Verdú E, Schargel Palacios K, et al. Modification of the conjuntival flora with cleaning palpebral solutions. Arch Soc Esp Oftalmol. 2004;79(12):617-621.

15. Stead RE, Stuart A, Keller J, Subramaniam S. Reducing the rate of cataract surgery cancellation due to blepharitis. Eye (Lond). 2010;24(4):742.

16. Gautheron P, Dukic M, Alix D, Sina JF. Bovine corneal opacity and permeability test: an in vitro assay of ocular irritancy. Fundam Appl Toxicol. 1992;18(3):442-449.

17. Marzulli FN, Maibach HI. Contact allergy: predictive testing in man. Contact Dermatitis. 1976;2:1-17.

18. Korb DR, Greiner JV. Increase in tear film lipid layer thickness following treatment of meibomian gland dysfunction. Adv Exp Med Biol. 1994;350:293-298.

19. Olson MC, Korb DR, Greiner JV. Increase in tear film lipid layer thickness following treatment with warm compresses in patients with meibomian gland dysfunction. Eye Contact Lens. 2003;29:96-99.

PubMed Central and CAS, and is the official journal of The Society of Clinical Ophthalmology (SCO). The manuscript management system is completely online and includes a very quick and fair peer-review system, which is all easy to use. Visit http://www.dovepress.com/ testimonials.php to read real quotes from published authors. 